\title{
Economic and Environmental Efficiency in Europe: evidence from a new Stochastic Frontier Model ${ }^{1}$
}

\author{
Margarita Robaina Alvesa, Victor Moutinho ${ }^{b}$, Pedro Macedoc \\ aGOVCOPP - Research Unit in Governance, Competitiveness and Public Policy, and Department of Economics, \\ Management and Industrial Engineering, University of Aveiro, Campus de Santiago, 3810-193, Aveiro, Portugal, \\ mrobaina@ua.pt \\ ${ }^{b}$ CEFAGE - Center of Advanced Studies in Management and Economics, University of Évora, Portugal and Department of \\ Economics, Management and Industrial Engineering, University of Aveiro, Campus de Santiago, 3810-193, Aveiro, \\ Portugal, vmoutinho@ua.pt \\ cCIDMA - Center for Research and Development in Mathematics and Applications, Department of Mathematics, \\ University of Aveiro, Campus de Santiago, 3810-193, Aveiro, Portugal, pmacedo@ua.pt
}

\begin{abstract}
This study aims to evaluate the resource and environment efficiency problem of European countries. We specify a new stochastic frontier model where Gross Domestic Product (GDP) is considered as the desirable output and Greenhouse Gases (GHG) emissions as the undesirable output. Capital, Labour, Fossil fuels and Renewable Energy consumption are regarded as inputs. The study is divided into two distinct periods, 2000-2004 and 2005-2011, in order to evaluate the difference between efficiency levels before and after the establishment of environmental targets related with the implementation of the Kyoto Protocol in 2005. A maximum entropy approach to assess technical efficiency is discussed.
\end{abstract}

Keywords: Eco-efficiency, European countries, Renewable energy, Stochastic frontier analysis

\section{Introduction}

Economic activities use production factors as energy resources, labour and capital to produce desirable goods and services, but simultaneously produce undesirable outputs, such as Greenhouse Gases (GHG), and particularly, Carbon Dioxide (CO2) emissions. Economic efficiency does not imply environmental efficiency, as the production processes may rely too much on fossil fuels or technologies, which although technically efficient, and cheap, lead to high levels of emissions or other environmental impacts. But if there is technical or economic inefficiency, it can cause environmental inefficiency. For example, waste of raw materials, or inefficient use of energy leads to a technical, economic and environmental inefficiency also because we are wasting resources and increasing pollution.

There are several ways to measure the so-called Eco-Efficiency (EE), which depend on the purpose and scope of the study. As defined by the World Business Council for Sustainable Development (WBCSD), "eco-efficiency is achieved by the delivery of competitively priced goods and services that satisfy human needs and bring quality of life, while progressively reducing ecological impacts and resource intensity throughout the life-cycle to a level at least in line with the Earth's estimated carrying capacity." The concept is concerned with creating more value with less impact (www.wbcsd.org). Other definitions of EE can be pointed, as "the efficiency with which ecological resources are used to meet human needs", by [1] or "the ability of firms, industries or economies to produce goods and services while incurring less impact on the environment and consuming fewer natural resources" by [2] .

${ }^{1}$ Final version of this work is published in the Proceedings of the $12^{\text {th }}$ International Conference on the European Energy Market (EEM), 2015, IEEE (DOI: 10.1109/EEM.2015.7216678; http://ieeexplore.ieee.org/xpl/articleDetails.jsp?arnumber=7216678). (C) 2015 IEEE. Personal use of this material is permitted. Permission from IEEE must be obtained for all other uses, in any current or future media, including reprinting/republishing this material for advertising or promotional purposes, creating new collective works, for resale or redistribution to servers or lists, or reuse of any copyrighted component of this work in other works. 
The simplest indicator of EE relates the economic output or Gross Domestic Product (GDP) with the environmental impact caused by the production process, for instance, the ratio GDP/CO2. As the production process may give rise to other environmental impacts, other measures, that replace $\mathrm{CO} 2$ by a composite good of environmental pressures, have emerged [3]. This study aims to evaluate the resource and environment efficiency (Eco-efficiency) problem of European countries. We specify a new stochastic frontier model where GDP is considered as the desirable output and GHG emissions as the undesirable output. We use the ratio between GDP and GHG emissions as definition of EE. Fossil fuel consumption, Renewable Energy Consumption, Capital and Labour are regarded as inputs. GDP by GHG emissions ratio is maximized given the values of the other four variables. The study is divided into two distinct periods: 2000-2004 and 2005-2011. This division is related to the implementation of the Kyoto Protocol in 2005, and will allow us to evaluate the difference between the levels of eco-efficiency before and after the establishment of environmental targets.

In this study, a stochastic frontier approach using some maximum entropy (ME) estimators is proposed as an alternative to the Kaya identity. A new maximum entropy approach to assess technical efficiency, which combines information from the data envelopment analysis (DEA) and the structure of composed error from the stochastic frontier analysis (SFA) without requiring distributional assumptions, is presented in this work. Technical efficiency was estimated, but as the maximized output is the GDP/GHG ratio, the estimation of technical efficiency is also a measure of eco-efficiency.

\section{Literature Review}

The use of benchmarking and activity analysis or DEA techniques have emerged in recent years as more sophisticated techniques to assess the EE of the countries and/or economic sectors. The use of DEA to do cross country and over time comparisons of EE has been used in various studies as in [4]-[6]. Some authors study sectoral EE, such as [2], [7]-[13]. Particularly at the macro level, there are scarce studies that analyze and evaluate the environmental and technical efficiency, particularly in the application of stochastic frontier parametric models. In light of this gap in the literature and the relevance of this topic, there is an urgent need to develop robust estimation techniques. In this study, the parametric stochastic frontier approach using some maximum entropy estimators, namely the generalized maximum entropy (GME) and the generalized cross-entropy (GCE) are proposed.

\section{Methodology}

Several methods to estimate technical efficiency are available in the efficiency literature, being DEA and SFA the most dominant methods. The stochastic frontier model in this work is estimated through maximum likelihood (ML), GME and GCE estimators. For reader's convenience, the ME principle and the GME estimator are briefly discussed next.

The ME formalism was first established by [15], [16] based on physics (Shannon entropy and statistical mechanics) and statistical inference. The ME principle provides a tool to make the best predictions from the limited available information. An interesting feature of ME is that it can be seen as an extension of Bernoulli's principle of insufficient reason; e.g., [15]. As noted by [14], statistical data are frequently limited and affected by collinearity implying that the associated statistical models may be ill-posed, unless simplifying assumptions or procedures are imposed to generate seemingly well-posed statistical models that can be estimated with traditional statistical tools. Giving heed to this problem, [14] generalized the ME formalism and developed the GME estimator, which is useful in models exhibiting collinearity, in models with small samples sizes and non-normal errors, as well as in models where the number of parameters to be estimated exceeds the number of observations available (under-determined models).

Considering the linear regression model defined in matricial form as

$$
\boldsymbol{y}=\boldsymbol{X} \boldsymbol{\beta}+\boldsymbol{e}
$$

where $\boldsymbol{y}$ denotes a $(N \times 1)$ vector of noisy observations, $\boldsymbol{\beta}$ is a $(K \times 1)$ vector of unknown parameters, $\boldsymbol{X}$ is a known $(N \times$ $K)$ matrix of explanatory variables and $\boldsymbol{e}$ is a $(N \times 1)$ vector of random errors, [14] treat each $\beta_{k}$ as a discrete random variable with a compact support and $M \geq 2$ possible outcomes, and each $e_{n}$ as a finite and discrete random variable with $J \geq 2$ possible outcomes. Assuming that both the unknown parameters and the unknown error terms may be bounded $a$ priori, the linear model can be presented as

$$
y=X Z p+V w
$$

where 


$$
\boldsymbol{\beta}=\boldsymbol{Z} \boldsymbol{p}=\left[\begin{array}{cccc}
\boldsymbol{z}_{1}^{\prime} & \mathbf{0} & \ldots & \mathbf{0} \\
\mathbf{0} & \mathbf{z}^{\prime}{ }_{2} & \ldots & \mathbf{0} \\
\vdots & \vdots & \ddots & \vdots \\
\mathbf{0} & \mathbf{0} & \ldots & \boldsymbol{z}^{\prime}{ }_{K}
\end{array}\right]\left[\begin{array}{c}
\boldsymbol{p}_{1} \\
\boldsymbol{p}_{2} \\
\vdots \\
\boldsymbol{p}_{K}
\end{array}\right]
$$

with $\boldsymbol{Z}$ a $(K \times K M)$ matrix of support values and $\boldsymbol{p}>\mathbf{0}$ a $(K M \times 1)$ vector of unknown weights, and

$$
\boldsymbol{e}=\boldsymbol{V} \boldsymbol{w}=\left[\begin{array}{cccc}
\boldsymbol{v}_{1} & \mathbf{0} & \ldots & \mathbf{0} \\
\mathbf{0} & \boldsymbol{v}_{2}^{\prime} & \ldots & \mathbf{0} \\
\vdots & \vdots & \ddots & \vdots \\
\mathbf{0} & \mathbf{0} & \ldots & \boldsymbol{v}_{N}^{\prime}
\end{array}\right]\left[\begin{array}{c}
\boldsymbol{w}_{1} \\
\boldsymbol{w}_{2} \\
\vdots \\
\boldsymbol{w}_{N}
\end{array}\right]
$$

with $\boldsymbol{V}$ a $(N \times N J)$ matrix of support values and $\boldsymbol{w}>\mathbf{0}$ a $(N J \times 1)$ vector of unknown weights. The GME estimator is given by

$$
\underset{\boldsymbol{p}, \boldsymbol{w}}{\operatorname{argmax}}\left\{-\boldsymbol{p}^{\prime} \ln \boldsymbol{p}-\boldsymbol{w}^{\prime} \ln \boldsymbol{w}\right\},
$$

subject to the model constraint,

$$
y=X Z p+V w,
$$

and the two additivity constraints for $\boldsymbol{p}$ and $\boldsymbol{w}$, respectively,

$$
\begin{aligned}
& \mathbf{1}_{K}=\left(\boldsymbol{I}_{K} \otimes \mathbf{1}_{M}^{\prime}\right) \boldsymbol{p}, \\
& \mathbf{1}_{N}=\left(\boldsymbol{I}_{N} \otimes \mathbf{1}_{J}^{\prime}\right) \boldsymbol{w},
\end{aligned}
$$

where $\otimes$ represents the Kronecker product. The GME estimator generates the optimal vectors $\widehat{\boldsymbol{p}}$ and $\widehat{\boldsymbol{w}}$ that can be used to form point estimates of the unknown parameters and the unknown errors through the reparameterizations defined previously. Additional details concerning the GME and the GCE estimators can be found in [14] and [17].

Recently, an increasing interest with the GME and GCE estimators in technical efficiency analysis has emerged in the literature; e.g., [18]-[21]. The main motivation comes from the advantages of the ME estimation which avoids criticisms and difficulties of DEA and SFA. For instance, with ME estimation the DEA method is used only to define an upper bound for the supports and thus the main criticism on DEA is used as an advantage. Furthermore, the composed error structure in SFA is used without distributional assumptions, which means that the main criticism on SFA is avoided with ME estimation. Thus, by avoiding criticisms and difficulties of DEA and SFA, the ME estimators appear to be a promising approach in efficiency analysis. Methodological details on the estimation of technical efficiency with GME and GCE estimators in this study can be found in [22].

\section{Results}

The closer the value of EE is from unit, the more efficient the country is, which means that the country is making the best use of resources to produce the maximum possible and at the same time is minimizing the environmental impact through GHG emissions. Regarding the ranking of countries, the different methods used to evaluate the EE show very similar results. Therefore we will focus on the analysis and interpretation of results concerning modification on the ranking of countries as well as in the trend of EE in the first and the second period (before and after the Kyoto Protocol commitment), considering only the results of the GCE estimator.

In the first period, before the Kyoto Protocol, according to Figure 1, the empirical evidence shows that Sweden, United Kingdom, Latvia, Cyprus and France are the five most efficient countries, while Estonia, Czech Republic and Greece constitute the least efficient countries. Furthermore, as shown in Table 1, Portugal and Slovenia were in the 20th and 17th position respectively, in 2000 and are ranked in 12th and 10th position on 2004. Conversely, Bulgaria and Italy were ranked at 8th and 10th position in 2000 and changed the ranking to 17th and 18th respectively in 2004. That evidence suggests that Portugal and Slovenia are becoming relatively more efficient, while Bulgaria and Italy became the least economic and environmental efficient countries at the end of the first period of analysis. 
For the second period analyzed (2005-2011), it can be seen from Figure 2, that Sweden, Latvia, UK, Hungary, Portugal and Cyprus are the six most efficient countries, while, Czech Republic, Poland and Estonia, constitute the three least efficient countries. According to Table 1, Hungary, Slovenia, Portugal and Ireland are ranked in 8th, 12th, 13th and 23rd place respectively in 2005 and change their ranking to 3rd, 5th, 4th and 7th place respectively in 2011. This suggests a significant change in the trend of economic and environmental efficiency. Conversely, Romania, Lithuania and Denmark are ranked in 4th, 9 th and 11th place respectively in 2005, and drop position to 11th, 13th and 17th respectively at the end of the period.

Figure 1. Eco-Efficiency estimates in 2000-2004 provided by the GCE estimator

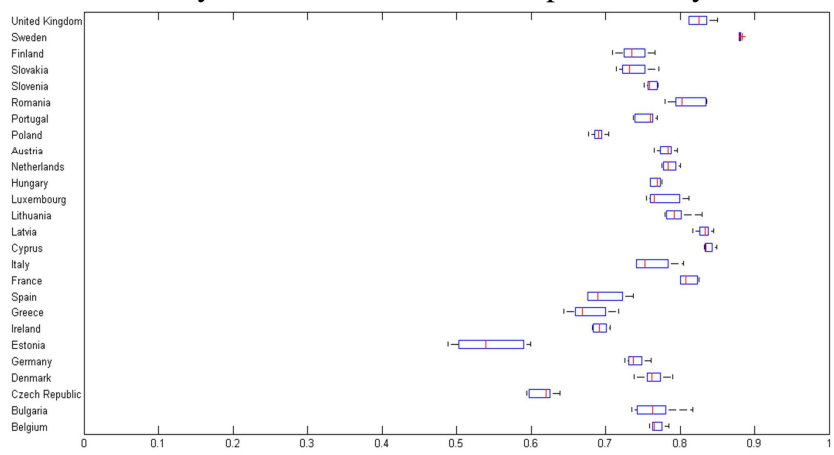

Table 1. Rankings of Eco-Efficiency in European Countries established by the GCE estimator

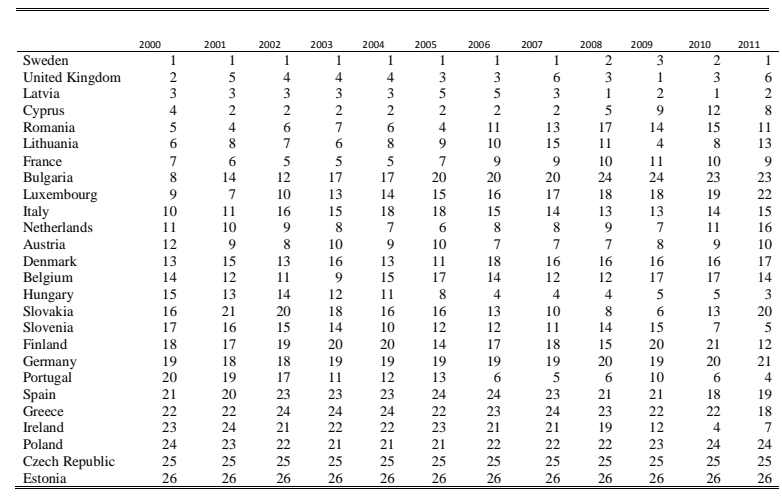

Figure 2. Eco-Efficiency estimates in 2005-2011 provided by the GCE estimator

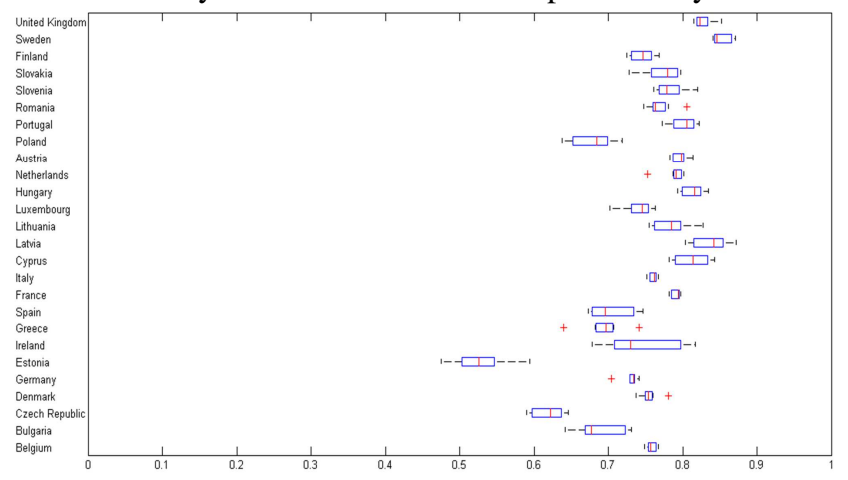

The EE estimates using the three estimation techniques (GME, GCE and ML) for these European countries show that changes in energy sources, capital and labour might give a reasonable simultaneous indication of the economic and environmental efficiency improvements. Analyzing the EE path we can point out some relevant facts. For the period as a whole, some countries that performed well, such as Ireland, which increased its level of EE by $50 \%$ (from 0.65 to 0.95 ), Hungary, by $23 \%$ (from 0.77 to 0.95 ), Portugal and Slovenia, by $22 \%$ (from 0.74 to 0.91 and 0.76 to 0.92 respectively). This good performance was particularly strong in the second period. On the other hand, Bulgaria and Estonia experienced a bad performance overall, by dropping the EE level of 0.83 to $0.53(-36 \%)$ and from 0.54 to $0.29(-47 \%)$ respectively. 


\section{Conclusions}

This study evaluated the Eco-efficiency problem of European countries in two distinct periods: 2000-2004 and 2005-2011. We specified a new stochastic frontier model where the ratio between GDP and GHG emissions is maximized given the values of Fossil Fuel Consumption, Renewable Energy Consumption, Capital and Labour. We could identify the change in the positioning of the countries in relation to EE in the two periods under review. The most efficient (Portugal, Slovakia, Hungary, Ireland) and the least efficient countries (Bulgaria, Italy, Romania, Denmark) were noted as well as a greater effort to converge to the frontier of efficiency by some countries in the second period of the analysis which coincides with the period after the ratification of the Kyoto Protocol. Since the ratification of the Kyoto Protocol, the European countries have taken various initiatives to reduce emissions and this was noted in the evolution of the level of eco-efficiency of some countries, particularly in the second period.

\section{Acknowledgments}

This work was partially supported by Portuguese funds through the CEFAGE (Center for Advanced Studies in Management and Economics), the GOVCOPP (Research Unit in Governance, Competitiveness and Public Policy), the CIDMA (Center for Research and Development in Mathematics and Applications), and the Portuguese Foundation for Science and Technology ("FCT - Fundação para a Ciência e a Tecnologia"), within project UID/MAT/04106/2013.

\section{References}

[1] OECD, Organization for Economic Co-operation and Development. Ecoefficiency, OECD, Paris, 1998.

[2] A. J. Picazo-Tadeo, J. A. Gómez-Limón and E. Reig-Martínez, "Assessing farming eco-efficiency: a data envelopment analysis approach", Journal of Environmental Management, vol. 92(4), pp. 1154-1164, 2011.

[3] S. Schmidheiny and J. L. Zorraquin, Financing Change, the Financial Community, Eco-Efficiency and Sustainable Development. Cambridge: MIT Press, 1996.

[4] F. Taskin and O. Zaim, "Searching for a Kuznets curve in environmental efficiency using kernel estimations", Economics Letters, vol. 68, pp. 217-223, 2000.

[5] O. Zaim and F. Taskin, “A Kuznets curve in environmental efficiency: an application on OECD countries", Environmental Resource Economics, vol. 17, pp. 21-36, 2000.

[6] K. Rashidi, A. Shabani and R. Saen, "Using data envelopment analysis for estimating energy saving and undesirable output abatement: a case study in the Organization for Economic Co-Operation and Development (OECD) countries", Journal of Cleaner Production, in press.

[7] A. J. Picazo-Tadeo, M. Beltrán-Esteve and J. A. Gómez-Limón, “Assessing eco-efficiency with directional distance functions", European Journal of Operational Research, vol. 220(3), pp. 798-809, 2012.

[8] A. J. Picazo-Tadeo and D. Prior, "Environmental externalities and efficiency measurement", Journal of Environmental Management, vol. 90(11), pp. 3332-3339, 2009.

[9] S. K. Mandal, "Do undesirable output and environmental regulation matter in energy efficiency analysis? Evidence from Indian cement industry", Energy Policy, vol. 38(10), pp. 6076-6083, 2010.

[10] Y. Barba-Gutiérrez, B. Adenso-Díaz and S. Lozano, "Eco-efficiency of electric and electronic appliances: a data envelopment analysis (DEA)", Environmental Modeling \& Assessment, vol.14, pp. 439-447, 2009.

[11] M. Kortelainen and T. Kuosmanen, "Measuring eco-efficiency of production: a frontier approach", EconWPA, Working Paper 0411004, Washington University, St. Louis, 2005.

[12] G. Egilmez, M. Kucukvar and O. Tatari, "Sustainability assessment of U.S. manufacturing sectors: an economic input output-based frontier approach", Journal of Cleaner Production, vol. 53, pp. 91-102, 2013.

[13] A. Avadí, I. Vázquez-Rowe and P. Fréon, "Eco-efficiency assessment of the Peruvian anchoveta steel and wooden fleets using the LCA+DEA framework", Journal of Cleaner Production, vol. 70, pp. 118-131, 2014.

[14] A. Golan, G. Judge and D. Miller, Maximum Entropy Econometrics: Robust Estimation with Limited Data. Chichester: Wiley, 1996.

[15] E. T. Jaynes, "Information theory and statistical mechanics", Physical Review, vol. 106(4), pp. 620-630, 1957.

[16] E. T. Jaynes, "Information theory and statistical mechanics. II", Physical Review, vol. 108(2), pp. 171-190, 1957.

[17] R. Mittelhammer, N. C. Cardell and T. L. Marsh, "The data-constrained generalized maximum entropy estimator of the GLM: asymptotic theory and inference", Entropy, vol. 15, pp. 1756-1775, 2013.

[18] R. Campbell, K. Rogers and J. Rezek, "Efficient frontier estimation: a maximum entropy approach", Journal of Productivity Analysis, vol. 30(3), pp. 213-221, 2008.

[19] J. Rezek, R. Campbell and K. Rogers, "Assessing total factor productivity growth in Sub-Saharan African agriculture", Journal of Agricultural Economics, vol. 62(2), pp. 357-374, 2011.

[20] P. Macedo and M. Scotto, "Cross-entropy estimation in technical efficiency analysis", Journal of Mathematical Economics, vol. 54 , pp. 124-130, 2014.

[21] P. Macedo, E. Silva and M. Scotto, "Technical efficiency with state-contingent production frontiers using maximum entropy estimators", Journal of Productivity Analysis, vol. 41(1), pp. 131-140, 2014

[22] M. Robaina-Alves, V. Moutinho and P. Macedo, "A new frontier approach to model the eco-efficiency in European countries", Journal of Cleaner Production, in press. 